\title{
Attitudes of dental students towards paediatric dental behaviour guidance
}

\author{
Maria Shindova ${ }^{1, *}$ iD, Ani Belcheva² (iD
}

${ }^{1}$ Senior Assistant Professor, Department of Pediatric Dentistry, Faculty of Dental Medicine, Medical University of Plovdiv, Bulgaria.

${ }^{2}$ Professor, Department of Pediatric Dentistry, Faculty of Dental Medicine, Medical University of Plovdiv, Bulgaria.
Corresponding author:

Maria Shindova, DDS, MSc, PhD Senior Assistant Professor Department of Pediatric Dentistry Faculty of Dental Medicine Medical University - Plovdiv 3 Hristo Botev Bvd 4000 Plovdiv, Bulgaria Mobile: + 359898390935 email: mariya.shindova@gmail.com Mariya.Shindova@mu-plovdiv.bg

Editor: Altair A. Del Bel Cury

Received: April 12, 2021

Accepted: August 24, 2021
Aim: To compare the pre-clinical and clinical students perceptions about the non-pharmacological behaviour management techniques in paediatric dentistry and to investigate the influence of the dental curriculum on the students' knowledge regarding this issue. Methods: A total of 283 students from the IV-and X-semester completed a questionnaire, consisted of 12 statements, describing the nonpharmacological behaviour management techniques for the treatment of paediatric dental patients. The acceptability rate was evaluated with a Likert scale ranging from 1 to 5 . Results: The students from all courses demonstrated high acceptance for Reinforcement and Desensitization techniques and low for the Negative reinforcement and Physical restraint. The comparison between the perceptions of the pre-clinical and clinical students demonstrated a statistically significant increase in the acceptance of the physical restraint, along with Nonverbal communication, Modelling and Parental presence/absence $(p<0.05)$. Conclusion: The results provide information about the students' knowledge and skills in behaviour management techniques together with some insights about how the educational process can modify the students' perceptions and views in dealing with paediatric dental patients.

Keywords: Students. Behavior. Methods. Pediatric dentistry. 


\section{Introduction}

One of the most challenging aspects in the dental practice is working with uncooperative patients and their behaviour management. Dentists are expected to be aware of the wide variety of behaviour management techniques (BMTs) and to use the most suitable one according to the individual clinical situation in order to provide adequate clinical care. In addition to this, dental practitioners should be tolerant and flexible in incorporating behaviour management strategies ${ }^{1}$. The American Academy of Pediatric Dentistry (AAPD) has issued a set of guidelines on behaviour guidance for paediatric dental patients with recommendations for focusing and implementing them during the entire period of dental education². It identifies both basic and advanced BMTs, as well as the indications for their use ${ }^{2}$. As to the advanced BMTs such as Positive stabilization, Hand over mouth, use of Papoose Boards, sedation and general anesthesia, it is recommended for use only for those dentists who have completed postdoctoral training 2,3 . While for the basic BMTs, students receive theoretical and clinical training during their dentistry training at the university. Dental education in paediatric dentistry should provide the opportunity for students to learn, observe, put into practice the different behaviour management strategies and techniques. Al-Jobair et al. considered that the content of the educational curriculum and the degree of the training in BMTs have an impact on the students ' perceptions and practice of such techniques ${ }^{4}$. There have been some empirical studies evaluating the acceptability of the BMTs during dental treatment. They can be divided into three approaches. The first one corresponds to the parent's views and attitudes with a child currently receiving dental treatment ${ }^{5-7}$. The second approach has compared how children faced these strategies ${ }^{8,9}$. And the third one has examined the views of individuals who have not had direct experience of the treatment ${ }^{10}$. Dental practitioners ' and students ' views are associated not only with the dental experience but also with the educational level during the learning process $^{10}$. To a large extent, dentist's perceptions about BMTs for the treatment of dental patients are based on the information obtained during their dental education and on the experience derived from the contact with patients ${ }^{11}$. At the beginning of the course of paediatric dentistry, students have no or limited knowledge of BMTs, thus their views could be compared with those of parents and the general public ${ }^{12}$. These perceptions are important and play a significant role in determining the acceptance by the public and their implementation ${ }^{12}$. Nevertheless, as they receive didactics classes about BMTs and start to provide treatment for children, their view may be changed by the influence of the educational process ${ }^{11}$.

Thus, the purpose of the present study was to compare the fourth- (pre-clinical) and tenth- (clinical) semester students' perceptions about the non-pharmacological BMTs in paediatric dentistry and to investigate the influence of the dental curriculum on the students' knowledge regarding this issue.

\section{Material and methods}

The study consisted of an anonymous, self-completed e-mailed survey. Potential subjects received an email describing the study and inviting their participation. A total of 460 students (250 students from the fourth semester (IInd course) and 210 
from the tenth semester ( $\mathrm{V}^{\text {th }}$ course) were invited to participate in the study. The mail included a brief cover letter explaining the purpose of the survey. The study was conducted in January 2021 (within three weeks) and consisted of 12 statements, describing the non-pharmacological BMTs for the treatment of paediatric dental patients. To obtain students' acceptability scores, they had to mark on a 5-point Likert scale from 1 to 5 (figure 1).

\section{Ethical principles}

The study is conducted in accordance with the conditions and principles of the Declaration of Helsinki, the existing EU Clinical Trial Directive (EC) No. 2001/20/EC, the recommendations of the Ethical Committee at the Medical University of Plovdiv, Bulgaria and the International ethical and scientific quality standard for designing, recording and reporting trials that involve the participation of human subjects - Good Clinical Practices (GCP).

The study was approved by the Committee for Scientific Research Ethics, Medical University - Plovdiv, Bulgaria (Reference number P-1371/30.04.2018, Protocol of approval No. 2/01.04.2021) before circulating the questionnaire.

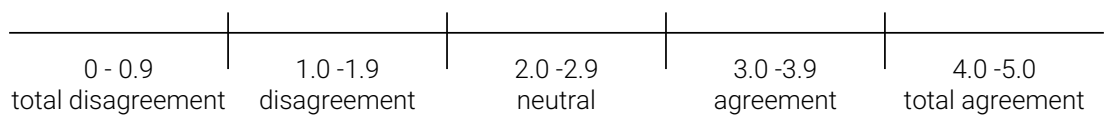

Figure1.Rating of acceptability to determine students ' perceptions

\section{Statistical analysis}

The obtained data were tabulated, processed and analysed using a SPSS (Statistical Package for Social Science software) version 21.0 (IBM, USA). Independent t-test was used to evaluate the statistically significant differences between the fourth-semester means and those obtained at the tenth-semester. The level of significance was set at $p<0.05$.

\section{Results}

Out of the 460 surveys that were e-mailed, 283 subjects (61.52\% response rate) were included in the statistical analysis for this study. The sample size was $N_{1}=131$ (for the fourth-semester) and $\mathrm{N}_{2}=152$ (for the tenth-semester). Table 1 shows the means and standard deviations of the acceptability scores as well as the statistical differences between the students' rates from the two investigated courses.

The most acceptable BMTs among the students from the two semesters were Nonverbal communication, Stop signals, Positive reinforcement (PR) and Tell-Show-Do (TSD). There were no changes in the levels of acceptability of these BMTs when the perceptions of the students from the two courses were compared ( $p>0.05)$, except for Nonverbal communication $(p=0.011)$. Considering the Desensitization techniques only the acceptability of Modelling increased significantly, changing from 'agreement’ to 'total agreement' $(p<0.01)$. 
The analysis of the views of all participants of our study showed that the least acceptable techniques were the aversive ones - Negative reinforcement (NR) and Restraint. Both showed a statistically significant increase in the scores of the group of clinical respondents $(p<0.001$ and $p=0.006)$. However, Voice control $(V C)$ as an aversive technique was defined as ‘acceptable` among the two investigated groups of students, no statistically significant difference was observed in the comparison ( $p>0.05)$.

Statistically significant differences in the acceptability rates concerning other BMTs were also found. The perceptions of Latent inhibition were 'acceptable' for the pre-clinical group and 'completely acceptable' for the clinical group of respondents $(p=0.003)$. As to the parent involvement in the dental treatment, the present results demonstrated that the Parental presence/absence technique became more acceptable throughout the semesters.

Table 1. Means and standard deviations of the acceptability scores and comparisons between students rates from the pre-clinical and clinical students

\begin{tabular}{lccc}
\hline \multirow{2}{*}{ BMT } & \multicolumn{2}{c}{ Means \pm SD } & Comparison \\
\cline { 2 - 4 } & Fourth semester & Tenth semester & P \\
\hline Nonverbal communication & $4.64 \pm .72$ & $4.81 \pm 0.42$ & $0.011^{*}$ \\
\hline Tell-Show-Do (TSD) & $4.44 \pm 0.67$ & $4.50 \pm 0.74$ & 0.533 \\
\hline Voice control (VC) & $3.32 \pm 1.24$ & $3.52 \pm 1.09$ & 0.174 \\
\hline Positive reinforcement (PR) & $4.51 \pm 0.70$ & $4.34 \pm 0.93$ & 0.063 \\
\hline Negative reinforcement (NR) & $1.89 \pm 1.13$ & $2.56 \pm 1.26$ & $0.000^{*}$ \\
\hline Distraction & $4.13 \pm 0.85$ & $4.15 \pm 0.87$ & 0.836 \\
\hline Stop signals & $4.55 \pm 0.81$ & $4.47 \pm 0.76$ & 0.397 \\
\hline Modelling & $3.72 \pm 0.94$ & $4.01 \pm 0.93$ & $0.008^{*}$ \\
\hline Desensitization & $3.82 \pm 0.95$ & $3.97 \pm 1.01$ & 0.197 \\
\hline Parental presence/absence & $3.20 \pm 1.19$ & $3.65 \pm 1.10$ & $0.001^{*}$ \\
\hline Latent inhibition & $3.90 \pm 1.05$ & $4.21 \pm 0.82$ & $0.003^{*}$ \\
\hline Restraint & $1.97 \pm 1.29$ & $2.38 \pm 1.30$ & $0.006^{*}$ \\
\hline
\end{tabular}

*statistically significant difference, $\mathrm{P}<0.05$

\section{Discussion}

The results of the present study showed that some of the students ' perceptions about several techniques changed significantly throughout the under-graduation program.

In line with other similar studies, most students demonstrated an acceptance of methods categorized as Reinforcement and Desensitisation techniques such as PR, TSD, Distraction, Communication regardless of their course in the university ${ }^{3,4,11-13}$. A significant increase in the acceptability of Nonverbal communication was observed in the views of the $\mathrm{V}^{\text {th }}$-course students. The technique is simple, easy to be remembered and applied, as well as communicative techniques are included as a first option in the behaviour guidance strategies of the dental curriculum. Another desensitizing BMT also demonstrated a significant positive change in the present study - Modelling. The 
effectiveness of using it in the management of a child's dental behaviour during the clinical training classes by students could be a possible reason for our results ${ }^{4}$.

In contrast to other basic techniques, not many students accepted aversive methods. The analysis of the current results demonstrated a significantly higher acceptability mean score amongst clinical year students for the investigated aversive BMTs, except for VC, in comparison with their pre-clinical counterparts. Similar results were noticed by Sotto et al. ${ }^{12}$, Al-Jaboir et al. ${ }^{4}$, Ali et al. ${ }^{13}$. In many countries, the use of physical restraint on dental patients is guided by protocols and guidelines, which highlights the need for careful consideration of patients' physical, physiological, psychological and medical conditions ${ }^{14}$. As to VC, students from the fourth and the tenth semesters reported comparable perceptions for its use in the treatment of paediatric dental patients. The present findings appeared to contrast to the results found by York et al. ${ }^{3}$ and Ali et al. ${ }^{13}$ who indicated a significant positive change throughout the semesters in the university.

Students initially disagreed with NR but their perceptions shifted significantly towards 'neutral' at the end of the course. This could be explained by achieving better patient cooperation during dental treatment using this BMT. Following the current trend in the dental profession for a loss of the aversive techniques and an increase in pharmacological management, future exploration of the perceptions about the pharmacological BMTs will be interesting.

Although Latent inhibition is time-consuming, requires attention to detail, more effort and special equipment in the dental office, this BMT became more acceptable throughout the semesters and interestingly more students reported using this technique with their patients ${ }^{3}$. In recent years, dental education and clinical practice implemented psychological principles and techniques into dental training. York et al. emphasized that cognitive-behavioural techniques, such as Latent inhibition, have become standard of patient care and were included in many dental curricula ${ }^{3}$.

In the present study, significantly higher acceptability scores were given by clinical students at the end of the dental course of education for Parental presence/absence. On one hand, they found that the involvement of the parent in the management and control of dental behaviour is useful during the treatment of patients in the clinical training classes. On the other hand, students followed the present clear trend for parents preferring to stay with their children during dental treatment ${ }^{15}$. Al-Jobair et al. ${ }^{4}$ also found that students' perceptions shifted significantly towards acceptance at the end of the educational process ${ }^{4,11}$. In contrast, the results of a study from 2020 demonstrated that significantly fewer Year 5 students, in comparison with students in Years 1 to 4, and amongst those in clinical years, accepted the presence of parents in the clinic during the procedure ${ }^{13}$.

The dental curriculum includes clinical training classes where students receive instructions for the use of different BMTs. Moreover, they have the opportunity to put them into practice and develop patient management skills ${ }^{4}$. The present findings indicated that dental education has adapted to the changing needs of patients and parents ${ }^{12}$. For future research, it is recommended the study be conducted on the same cohort of students to investigate the impact of education on their acceptance of various BMTs as well as the factors that influence such acceptance. 
In conclusion, there were significant differences in students' acceptance of Modelling and the aversive techniques-across academic years, between pre-clinical and clinical groups of respondents. Students' acceptance of the various techniques provides useful information for the Faculty of Dental Medicine about the students' knowledge and skills in BMTs, as well as it is an indication about how and to what extent this educational material can be modified in dental programmes.

\section{Data availability}

Datasets related to this article will be available upon request to the corresponding author.

\section{Conflicts of interests: none}

The investigators have no conflicts of interest to declare. They agree with the study and there is no financial interest to report.

\section{Acknowledgements}

The authors would like to show their gratitude to all students for participating in the survey.

\section{Funding}

This research will receive no specific grant from any funding agency in the public, commercial or not-for-profit sectors.

\section{Reference}

1. Feigal RJ. Guiding and managing the child dental patient. J Dent Educ. 2001 Dec;65(12):1369-77.

2. American Academy of Pediatric Dentistry. Behavior guidance for the pediatric dental patient. The Reference Manual of Pediatric Dentistry. Chicago, III.: American Academy of Pediatric Dentistry; 2020:292-310.

3. York KM, Mlinac ME, Deibler MW, Creed TA, Ganem I. Pediatric behavior management techniques: a survey of predoctoral dental students. J Dent Educ. 2007 Apr;71(4):532-9.

4. Al-Jobair AM, Al-Mutairi MA. Saudi dental students' perceptions of pediatric behavior guidance techniques. 2015 Sep 10;15:120. doi: 10.1186/s12909-015-0382-6.

5. Luis de León J, Guinot Jimeno F, Bellet Dalmau LJ. Acceptance by Spanish parents of behaviour-management techniques used in paediatric dentistry. Eur Arch Paediatr Dent. 2010 Aug;11(4):175-8. doi: 10.1007/BF03262739.

6. Elango I, Baweja DK,Shivaprakash PK. Parental acceptance of pediatric behaviour management techniques: a comparative study. J Ind Soc Pedod Prev Dent.2012;30:195-200.

7. Peretz B, Kharouba J, Blumer S. Pattern of parental acceptance of management techniques used in pediatric dentistry. J Clin Pediatr Dent. 2013 Fall;38(1):27-30.

8. Davies EB, Buchanan H. An exploratory study investigating children's perceptions of dental behavioural management techniques. Int J Paediatr Dent. 2013 Jul;23(4):297-309. doi: 10.1111/ipd.12007.

9. Kantaputra PN, Chiewcharnvalijkit K, Wairatpanich K, Malikaew P, Aramrattana A. Children's attitudes toward behavior management techniques used by dentists. J Dent Child (Chic). 2007;74(1):4-9.

10. Newton JT, Sturmey P. Students' perceptions of the acceptability of behaviour management techniques. Eur J Dent Educ. 2003 Aug;7(3):97-102. 
11. Oliveira R, Angelo AC, Brito DB, de Medeiros RC, Forte FD, Sousa SA. Student's Perceptions about Pediatric Dental Behavior Guidance Techniques throughout a five-year Dental Curriculum. Pesq Bras Odontopediatr Clin Integrada. 2015 Jun;15(1):143-52. doi: 10.4034/PBOCI.2015.151.16.

12. Sotto JJ, Azari AF, Riley J, Bimstein E. First-year students' perceptions about pediatric dental behavior guidance techniques: the effect of education. J Dent Educ. 2008;72(9):1029-41.

13. Ali NM, Husin IN, Ahmad MS, Hamzah SH. Perceptions of behavioural guidance techniques for paediatric patients amongst students in a Malaysian dental school. Eur J Dent Educ. 2021 Feb;25(1):18-27. doi: 10.1111/eje.12573.

14. Stirling $\mathrm{C}$, West M, editors. Guidelines for 'clinical holding' skills for dental services for people unable to comply with routine oral health care. British Society for Disability and Oral Health; 2009.

15. Vishwakarma AP, Bondarde PA, Patil SB, Dodamani AS, Vishwakarma PY, Mujawar SA. Effectiveness of two different behavioral modification techniques among 5-7-year-old children: A randomized controlled trial. J Indian Soc Pedod Prev Dent. 2017 Apr-Jun;35(2):143-9. doi: 10.4103/JISPPD.JISPPD_257_16. 